\title{
Avaliação dos requisitos da faixa de pista em aeroportos regionais: uma análise de risco
}

\author{
Helen Feuser Fernandes ${ }^{1}$, Carlos Müller ${ }^{2}$, Cláudio Jorge Pinto Alves ${ }^{3}$ \\ 1Instituto Tecnológico de Aeronáutica, helen.fernandes@gmail.com \\ 2Instituto Tecnológico de Aeronáutica, cmuller1954@gmail.com \\ 3Instituto Tecnológico de Aeronáutica, claudioj@ita.br
}

\section{Recebido:}

1 de outubro de 2017

Aceito para publicação:

13 de março de 2018

Publicado:

31 de dezembro de 2018

Editor de área:

Li Weigang

\section{Palavras-chaves:}

Aeroportos regionais,

Análise de risco,

Faixa de pista.

\section{Keywords:}

Regional airports,

Risk analysis,

Runway strip.

DOI:10.14295/transportes.v26i4.1478

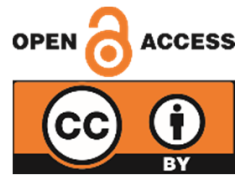

\begin{abstract}
RESUMO
O Brasil é o segundo país com maior número de aeródromos do mundo, atrás apenas dos Estados Unidos. Isso se deve principalmente ao grande número de aeroportos de pequeno porte existentes no país. Devido à dificuldade em se adequar às exigências impostas, esse trabalho tem como objetivo avaliar os requisitos exigidos para a faixa de pista em aeroportos regionais, de modo a viabilizar as operações nesses aeroportos. Para isso foi utilizado um modelo de análise de risco denominado ACRP 50 - Airport Cooperative Research Program, o qual foi aplicado ao Aeroporto de Joinville. Esse modelo foi criado através de uma base histórica de dados operacionais e meteorológicos, e calcula a probabilidade de a aeronave exceder os limites da pista. A partir do modelo, foram discutidas algumas das variáveis que influenciam as operações em aeroportos regionais. Além disso, o nível de segurança da faixa de pista do aeroporto foi avaliado e foram discutidas as implicações de alterações das dimensões da faixa de pista.
\end{abstract}

\section{ABSTRACT}

Due to the great number of small airports, Brazil has the second largest number of airfields, which is only overcome by the USA. Considering the difficulty in adapting to the imposed requirements, this work aims to evaluate the requirements for the runway strip of regional airports, in order to make feasible the operations on this kind of airport. For this purpose, a risk assessment model named ACRP 50 - Airport Cooperation Research Program was applied to the Joinville Airport. This model was developed by means of an operational and meteorological database and evaluates the runway excursion probability. Based on the model, a discussion is presented on the most influential variables for the operations on regional airports. In addition, the runway strip safety was assessed for the Joinville Airport, and it was discussed the implications of dimensional changes in the runway strip.

\section{INTRODUÇÃO}

O RBAC é o Regulamento Brasileiro da Aviação Civil, e ele está baseado no Anexo 14: Volume I - Aeródromos, definido a partir da Convenção sobre Aviação Civil Internacional (ANAC, 2009). Ambos trazem diretrizes para projetar as pistas de pouso e decolagem, além de apresentarem diretrizes para os demais componentes geométricos, tais como: faixa de pista, zona de parada stopway, zona desimpedida - clearway, pista de rolamento - taxiway, entre outros. Sendo que as regras para o dimensionamento dos componentes geométricos de um aeródromo são as mesmas tanto para aeroportos de grande porte quanto para os de pequeno porte.

Os aeroportos regionais oferecem voos regulares para as capitais e são usualmente interligados aos principais aeroportos através de um sistema denominado hub and spoke. Os principais aeroportos são os hubs que são conectados às demais regiões - centros menores chamadas de spokes. Segundo os autores Donehue e Baker (2012), os voos dos hubs aos aeroportos regionais 
são caracterizados por menor quantidade de passageiros e distâncias menores. A importância dos aeroportos regionais está relacionada ao atendimento emergencial de comunidades distantes. Halpern e Bråthen (2011) analisaram dois aeroportos na Noruega, um regional e outro de tamanho mediano com viagens nacionais e internacionais. Os autores observaram que os maiores propósitos das viagens nos dois aeroportos eram resultantes de trabalho e visita de parentes e amigos. No entanto, no que se refere à saúde, o aeroporto regional, localizado em uma região remota com menores recursos, apresentou maior procura. Outro exemplo da importância de aeroportos de pequeno porte é a Austrália que, de maneira similar ao Brasil, possui uma extensão territorial ampla e, portanto, necessita de aeroportos locais, equivalentes aos aeroportos regionais, para atender a crescente demanda do transporte aéreo.

Apesar da necessidade da aviação regional para suprir a demanda de transporte aéreo em todo o país, os acidentes da aviação civil no Brasil estão concentrados nos segmentos da aviação de menor porte: particular, instrução e aviação agrícola (Almeida et al., 2016). Esses segmentos correspondem a 77,8\% do total de acidentes registrados, considerando aeronaves de matrícula nacional, no período de 2006 a 2015 (Almeida et al., 2016). Embora as estatísticas apontem que existe maior probabilidade de acidentes e de incidentes ocorrerem no setor da aviação civil que opera em aeroportos de pequeno porte, o presente estudo questiona a necessidade de esses aeroportos seguirem as mesmas restrições operacionais dos principais aeroportos brasileiros e internacionais.

Esse questionamento baseia-se na dificuldade que alguns sítios aeroportuários têm em cumprir as normas da ICAO - International Civil Aviation Organization para segurança da pista, e pela grande quantidade de aeródromos de pequeno porte existentes no Brasil. Essa discussão se torna relevante no contexto brasileiro onde existem em torno de 2.000 aeródromos, entre públicos e privados, sendo que menos de 70 deles possuem mais de 1.000 movimentos de aeronaves por ano (INFRAERO, 2017). Recentemente noticiou-se que há uma tentativa, por parte da ANAC, em flexibilizar a norma que exige a permanência do carro dos bombeiros em aeroportos com menos de 200 mil passageiros por ano, tal como apontado no estudo de Freire (2016). Possíveis alterações na norma ajudariam a estimular o crescimento da aviação civil.

No entanto, qualquer alteração necessita de estudos que avaliem as consequências dessas mudanças. Os estudos aeronáuticos são exigidos no caso de incapacidade do cumprimento das normas ou das práticas recomendadas, sendo necessário demonstrar que o desvio existente não afeta a segurança operacional e o funcionamento regular das operações (DAC, 2004). Tais estudos devem ser realizados prioritariamente por meio de análise de risco, no entanto, na ausência de dados históricos que proporcionem uma análise estatisticamente significante recomenda-se o uso de um painel de especialistas. Os múltiplos fatores relacionados à ocorrência de um evento dificultam a caracterização das causas do evento, assim como informações incompletas na coleta de dados dessas ocorrências prejudicam a construção de novos modelos, que sejam mais precisos e adequados às análises estudadas (Wilke, Majumdar e Ochieng, 2015).

Para a condução de uma análise técnica da situação atual do aeroporto é necessário seguir três etapas: levantamento de dados; seleção dos procedimentos, métodos e fontes de dados; e análise de risco existente (DAC, 2004). Conforme sugerido pelo DAC - Departamento de Aviação Civil (2004), na primeira etapa são colhidas informações sobre as características do aeroporto - movimentação atual, mix de aeronaves, taxa de utilização das cabeceiras, condições topográficas e obstáculos na área do aeroporto, histórico de acidentes etc. Na segunda etapa são definidas as metodologias para a análise de risco, o impacto na capacidade operacional, nos custos e 
benefícios. Na última etapa é realizada a identificação da não conformidade existente no aeroporto, eventos indesejados são classificados e caracterizados, calcula-se o risco atual, a probabilidade de ocorrência e as consequências relativas a eventuais não conformidades (DAC, 2004).

A análise de risco envolve estimar o nível de risco existente, através de elementos que expliquem a ocorrência de possíveis eventos, e comparar os resultados com os critérios previamente estabelecidos (DAC, 2004). Após isso, deve-se decidir se o nível de risco é aceitável. 0 conceito de risco está relacionado às potenciais perdas envolvidas, sendo necessário avaliar a frequência, as consequências decorrentes dela, e a percepção de risco dos envolvidos (DAC, 2004). No caso de aeroportos, análises de risco em aeroportos têm sido conduzidas com objetivos diversos, tais como: estimar o nível de segurança operacional em relação a diferentes configurações de pistas (Barnett et al., 2015), determinar as taxas de acidentes na aviação geral relacionadas a regiões montanhosas e altitudes elevadas (Aguiar, Stolzer e Boyd, 2017), analisar o impacto das características dos aeroportos nas ocorrências de eventos (Wilke, Majumdar e Ochieng, 2015), dentre outros.

O modelo ACRP 50, desenvolvido através de uma parceria entre a FAA - Federal Aviation Administration e o TRB - Transportation Research Board, tem sido usualmente utilizado para este fim, principalmente devido ao histórico de dados utilizados. Este modelo estima a probabilidade de a aeronave sair dos limites laterais e longitudinais da pista, tanto para operações de pouso como de decolagem. Fortes e Correia (2012) e Correia e Ribeiro Neto (2014) aplicaram o modelo como metodologia de análise de risco em aeroportos brasileiros, com o objetivo de analisar o risco das operações de acordo com os obstáculos próximos a pista de pouso e decolagem. Os resultados obtidos por ambos os trabalhos foram condizentes com a realidade, ainda que em análise feita apenas para aeroportos de maior porte. Valdés et al. (2011) calcularam, através de uma versão anterior do modelo ACRP 50, possíveis flexibilizações quanto a geometria do lado ar do Aeroporto de La Palma na Espanha. Os autores buscavam alternativas para atender aos requisitos operacionais impostos em um sítio aeroportuário limitado pela geografia local. Com base no mesmo modelo, Trucco, De Ambroggi e Leva (2015) avaliaram a probabilidade de ultrapassagem dos limites da pista com base na energia cinética das aeronaves. Os resultados por eles encontrados são importantes para análises de viabilidade em torno do aeroporto, gerenciamento de segurança e futuras instalações do sítio aeroportuário.

Neste contexto, o presente estudo efetua uma análise de risco do Aeroporto de Joinville Lauro Carneiro de Loyola, SBJV/JOI, a fim de avaliar a possibilidade de diminuir as dimensões da faixa de pista de aeroportos regionais. 0 estudo é destinado, principalmente, a aeroportos regionais que estão localizados em áreas remotas, e que possuem baixa movimentação operacional. Isto é feito com base na exigência de os requisitos de segurança serem os mesmos, independente do tipo de operação, tipo de aeronave e tamanho do aeroporto. Devido à dificuldade em se obter os dados necessários para o desenvolvimento de um modelo, no presente estudo a análise de risco é realizada utilizando o modelo ACRP 50 (Ayres Jr. et al., 2011). Por se tratar de um modelo já utilizado na literatura para esse fim, inclusive em outros aeroportos brasileiros (Fortes e Correia, 2012; Correia e Ribeiro Neto, 2014), o artigo pressupõe a validade do modelo para iniciar uma discussão sobre os requisitos de segurança dos aeroportos regionais. Além de calcular a probabilidade de a aeronave sair dos limites da pista, o presente trabalho discute alguns dos fatores que influenciam as operações de pouso e decolagem em aeroportos de pequeno porte - regionais. Finalmente, os resultados da análise de risco são utilizados para avaliar se as dimensões atuais da faixa de pista do aeroporto em estudo podem ser diminuídas, o que 
resultaria em um menor custo na construção de novos aeródromos. Assim, este trabalho incita uma discussão, ainda primária, da possibilidade e da necessidade de alteração das normas vigentes para o dimensionamento de aeroportos, ou da criação de normas complementares específicas para aeroportos regionais.

Na Seção 2 deste trabalho apresenta-se o modelo de análise de risco, assim como as equações que o integram. Na Seção 3 são discutidas as variáveis que influenciam diretamente o comportamento das operações da aviação regional. Na Seção 4 são apresentados os dados de entrada, e as considerações admitidas para aplicação do modelo. Os resultados são apresentados e debatidos na Seção 5 e, finalmente, é apresentada a conclusão do trabalho.

\section{MODELO}

O modelo ACRP 50 (Ayres Jr. et al., 2011) foi criado com base em um histórico de dados, operacional e meteorológico, de países como Austrália, Inglaterra, Nova Zelândia, Singapura, Indonésia, Canadá, Estados Unidos, entre outros. No entanto, para o cálculo da frequência de incidentes, em específico, foram utilizados apenas dados de aeroportos dos Estados Unidos devido à ausência de dados dos demais países. Os acidentes e incidentes são de aeronaves que ultrapassaram as extremidades da pista em até 2000 pés, aproximadamente 600 metros, e de 1000 pés, aproximadamente 300 metros, do eixo central da pista. Aqui são analisados cinco tipos de incidentes, sendo que dois deles podem ocorrer na decolagem e três no pouso. Para a ocorrência de um incidente, deve-se analisar a ocorrência de vários fatores, sendo que a presença de algum ou a junção de vários desses fatores pode aumentar ou diminuir a probabilidade de o incidente analisado ocorrer. Os incidentes analisados são:

LDOR - Ultrapassagem no pouso - Landing Overrun;

LDUS - Toque antecipado no pouso - Landing Undershoot;

LDVO - Desvio lateral no pouso - Landing Veer-off;

TOOR - Ultrapassagem na decolagem - Take-off Overrun;

TOVO - Desvio lateral na decolagem - Take-off Veer-off.

Para o cálculo da probabilidade de ocorrer uma saída de pista, o modelo ACRP 50 (Ayres Jr. et al., 2011) utiliza a Equação (1):

$$
P\{\text { Ocorrênciade incidente }\}=\frac{1}{1+e^{\left(b_{0}+b_{1} x_{1}+b_{2} x_{2}+b_{3} x_{3}+\ldots+b_{n} x_{n}\right)}}
$$

onde $\quad \mathrm{P}\{$ Ocorrência de incidente\}: $\quad$ probabilidade de ocorrência de algum tipo de evento; Xi: $\quad$ Variáveis independentes - ex.: tipo de aeronave, visibilidade, chuva etc.;

$b_{i}$ : Coeficientes da regressão

Na Tabela 1 são apresentados os coeficientes e as variáveis do modelo para o cálculo de probabilidade de ocorrência de um dos cinco tipos de incidentes analisados. As operações no aeródromo são calculadas individualmente, de acordo com as características operacionais e meteorológicas do momento do pouso ou da decolagem. Quando determinado fator está presente, o coeficiente da variável é multiplicado pela variável independente $x_{1}$ que recebe o valor 1 , e é somado às demais variáveis presentes. Quando algum fator não corresponde às características do momento da operação, a variável independente $\mathrm{X}_{1}$ recebe o valor de zero. As magnitudes dos coeficientes são diferentes, indicando que alguns fatores influenciam mais do que outros em determinado evento, sendo que os coeficientes com sinais positivos aumentam a ocorrência, e os coeficientes com sinais negativos diminuem a probabilidade de acontecer o incidente. 
Tabela 1: Coeficientes e variáveis independentes para o modelo de probabilidade de incidente (Adaptado de Ayres Jr. et al., 2011)

\begin{tabular}{|c|c|c|c|c|c|c|}
\hline & & & & & & \\
\hline & Variável & LDOR & LDUS & LDVO & TOOR & TOVO \\
\hline & Constante ajustada & $-13,065$ & $-15,378$ & $-13,088$ & $-14,293$ & $-15,612$ \\
\hline \multirow{3}{*}{ Classe de Utilização } & $\mathrm{F}$ & & 1,693 & & 1,266 & \\
\hline & G & 1,539 & 1,288 & 1,682 & & 2,094 \\
\hline & $\mathrm{T} / \mathrm{C}$ & $-0,498$ & 0,017 & & & \\
\hline \multirow{2}{*}{ Classe da Aeronave } & $A / B$ & $-1,013$ & $-0,778$ & $-0,770$ & $-1,150$ & $-0,852$ \\
\hline & $D / E / F$ & 0,935 & 0,138 & $-0,252$ & $-2,108$ & $-0,091$ \\
\hline \multirow{3}{*}{ Nível do Teto } & menor que $200 \mathrm{ft}$ & $-0,019$ & 0,070 & & 0,792 & \\
\hline & 200 a $1000 \mathrm{ft}$ & $-0,772$ & $-1,144$ & & $-0,114$ & \\
\hline & 1000 a $2500 \mathrm{ft}$ & $-0,345$ & $-0,721$ & & & \\
\hline \multirow{3}{*}{ Visibilidade (Statute Mile) } & menor que $2 \mathrm{SM}$ & 2,881 & 3,096 & 2,143 & 1,364 & 2,042 \\
\hline & de 2 a 4 SM & 1,532 & 1,824 & & $-0,334$ & 0,808 \\
\hline & de 4 a 8 SM & 0,200 & 0,416 & & 0,652 & $-1,500$ \\
\hline \multirow{3}{*}{ Vento de Través } & de 2 a 5 nós & $-1,342$ & $-0,698$ & $-0,091$ & $-1,045$ & \\
\hline & de 5 a 12 nós & $-0,913$ & $-0,295$ & 0,653 & $-0,695$ & 0,102 \\
\hline & mais que 12 nós & $-0,921$ & $-1,166$ & 2,192 & 0,219 & 0,706 \\
\hline \multirow{2}{*}{ Vento de Cauda } & de 5 a 12 nós & & & 0,066 & & \\
\hline & mais que 12 nós & 0,786 & & 0,980 & & \\
\hline \multirow{3}{*}{ Temperatura } & menor que $5^{\circ} \mathrm{C}$ & 0,043 & 0,197 & 0,558 & 0,269 & 0,988 \\
\hline & de 5 a $15^{\circ} \mathrm{C}$ & $-0,019$ & $-0,71$ & $-0,453$ & $-0,544$ & $-0,42$ \\
\hline & Mais que $25^{\circ} \mathrm{C}$ & $-1,067$ & $-0,463$ & 0,291 & 0,315 & $-0,921$ \\
\hline \multirow{7}{*}{ Condições Meteorológicas } & Condições de gelo & 2,007 & 2,703 & 2,670 & 3,324 & \\
\hline & Chuva & & 0,991 & $-0,126$ & 0,355 & $-1,541$ \\
\hline & Neve & 0,449 & $-0,25$ & 0,548 & 0,721 & 0,963 \\
\hline & Granizo & & & $-0,103$ & & \\
\hline & Rajadas de vento & & 0,041 & $-0,036$ & 0,006 & \\
\hline & Neblina & & & 1,740 & & \\
\hline & Tempestade & $-1,344$ & & & & \\
\hline \multirow{5}{*}{ Fatores de Operação } & Turbo prop & & & $-2,517$ & 0,560 & 1,522 \\
\hline & Origem/Destino Internacional & 0,929 & 1,354 & $-0,334$ & & $-0,236$ \\
\hline & Aeroporto hub/não hub & 1,334 & & & & $-0,692$ \\
\hline & Fator de criticidade & 9,237 & 1,629 & 4,318 & & 1,707 \\
\hline & Condições noturnas & & & $-1,360$ & & \\
\hline
\end{tabular}

Após calcular a probabilidade de a aeronave ultrapassar os limites da pista, onde são obtidos valores entre 0 e 100\%, calcula-se a probabilidade da aeronave exceder determinada distância "d" desses limites. A Equação (2) apresenta a forma genérica para esse cálculo, e na Tabela 2 são apresentadas as equações para calcular a probabilidade de a aeronave exceder determinadas distâncias de acordo com cada tipo de evento - LDOR, LDUS, LDVO, TOOR, TOVO.

$$
P(d>t)=e^{-a t^{n}}
$$

onde $t$ : é a direção $\mathrm{X}$ - longitudinal a pista ou Y - transversal à pista que está sendo analisada; 
$P(d>t)$ : a probabilidade que alguma aeronave irá exceder certa distância " $\mathrm{d}$ " da pista, na direção analisada;

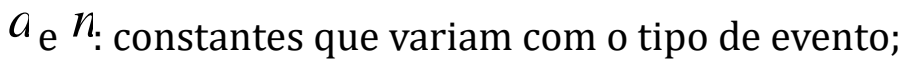

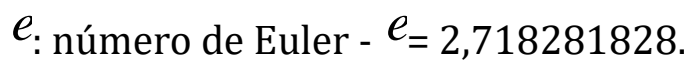

Tabela 2: Resumo dos modelos de probabilidade de localização (adaptado de Ayres Jr. et al., 2011)

\begin{tabular}{lll}
\hline Tipo de Acidente & Tipo de Dado & Modelo \\
\hline LDOR & Y & $P\{d>x\}=e^{-0,00321} x^{0,984941}$ \\
\hline LDUS & Y & $P\{d>y\}=e^{-0,20983 y^{0,4862}}$ \\
\hline LDVO & Y & $P\{d>y\}=e^{-0,02159 y^{0,773896}}$ \\
\hline TOOR & $P\{d>y\}=e^{-0,02568 y^{0,803946}}$ \\
\hline Y & $P\{d>x\}=e^{-0,00109 x^{1,06764}}$ \\
\hline
\end{tabular}

Logo, o resultado para cada operação fica como o apresentado na Equação 3. Leia-se o resultado como a probabilidade da aeronave exceder determinada distância nos eixos x e y na ocorrência de um evento. A Equação 3 descreve a multiplicação entre a Equação 1 e as equações de localização - Tabela 2.

$$
\text { Resultado }=P\{\text { Ocorrência de incidente }\} \cdot P\{d>x\} \cdot P\{d>y\}
$$

Note que quando o evento refere-se ao veer-off, LDVO e TOVO, o cálculo é efetuado apenas para o eixo y, pois trata da saída lateral da aeronave. 0 ponto de referência do veer-off é diferente do undershoot e do overrun, sendo o primeiro localizado na borda da pista $\mathrm{Y}^{*}$, e no caso dos dois seguintes, a partir do eixo da pista - $Y$ (Ayres Jr. et al., 2011). As equações da Tabela 2 já preveem esses pontos de referência distintos.

\section{VARIÁVEIS DO MODELO}

Apesar de o modelo conter uma base de dados de países que possuem clima e um histórico de operações diferentes entre si, as variáveis do modelo em conjunto, possibilitam a caracterização do comportamento da aviação civil quanto aos aspectos relacionados às saídas de pista. Nesse item são discutidas algumas das variáveis do modelo - Tabela 1, e suas influências na aviação regional para a ocorrência de incidentes.

\subsection{Classe da aeronave}

A classe da aeronave refere-se a uma classificação de tamanho de acordo com o máximo peso 
de decolagem, MTOW - Maximum Takeoff Weight. Quanto maior a aeronave, maiores são as exigências para sua utilização. Os requisitos impostos aos pilotos de aeronaves de grande porte são maiores do que para os pilotos que operam aeronaves menores (ANAC, 2012). Maiores exigências na aviação civil auxiliam na diminuição dos erros nas tomadas de decisões, evitando incidentes, diminuindo a probabilidade de ocorrência do fator "erro humano" como causa.

Em um estudo sobre acidentes na categoria de aviação geral, Bazargan e Guzhva (2011) afirmaram que quanto menor a experiência do piloto maior a taxa de acidentes tendo como causa o erro do piloto. Para medir a experiência do piloto, utilizou-se a quantidade de horas voadas como uma variável proxy - variável substituta que está relacionada, mas não é idêntica a variável direta, e ajuda a explicar a variável em caso de difícil mensuração (Wooldridge, 2009). Não apenas o quesito "competência" do piloto que opera aeronaves de grande porte é maior, mas também as exigências de manutenção e segurança de tráfego. Esses requisitos procuram cercar todo tipo de possibilidade de acidentes, sendo o fator "erro humano" o maior contribuinte para a causa deles (Almeida et al., 2016; Bandeira, Correia e Martins, 2017).

\subsection{Ventos}

O vento e suas características são fatores que definem a direção de operação da pista, devido ao cálculo do fator de utilização - tempo no qual a pista e seus componentes não ficam com as operações limitadas pelo vento de través e dos obstáculos no entorno do aeródromo (ANAC, 2009). De acordo com a direção de incidência em relação à pista/aeronave, os ventos podem ser denominados em três tipos: vento de través, vento de proa e vento de cauda. 0 vento de través é aquele que ocorre perpendicularmente à pista/aeronave e tem como característica desalinhar a trajetória da aeronave, efeito que é acentuado em condições de pista molhada. Cada aeronave possui um desempenho diferente, não devendo ser ultrapassado o limite de vento de través indicado no manual de voo.

0 vento de proa é aquele que tem sentido contrário ao da movimentação da aeronave, auxiliando as operações de pouso e decolagem. Na decolagem, devido ao formato das asas, ele ajuda a aeronave a levantar voo, já no pouso, acaba ajudando a frenagem. Por sua vez, o vento de cauda, ou vento de popa, é aquele que tem o mesmo sentido de deslocamento da aeronave, este último é útil no voo de cruzeiro, pois, tende a gerar economia de combustível e de tempo. Porém, para operações de pouso e decolagem esse vento é prejudicial, pois dificulta a frenagem da aeronave no pouso e prejudica o efeito da sustentação durante a corrida na decolagem, requerendo maiores distâncias a serem percorridas. De modo geral, a pista deve ser dimensionada com o objetivo de obter a maior incidência de vento de proa e menor vento de través. Outro fenômeno importante a ser analisado são as rajadas de vento - mudança repentina na intensidade e direção do vento, já que estes são considerados como um dos maiores causadores de danos (INMET, 2015).

\subsection{Turboprop}

As fases de pouso e decolagem demandam um esforço acentuado do piloto, com ênfase para o pouso. No pouso, grande parte da energia antes utilizada deve ser dissipada a fim de diminuir a distância a ser percorrida na pista. Os turboélices são aviões mais lentos que os jatos. Já os jatos possuem o agravante de serem, na maioria das vezes, aeronaves maiores, mais pesadas e, portanto, com maior energia. Ventos de través mais intensos afetam mais a manobrabilidade de aeronaves leves, se comparado às aeronaves mais pesadas, sendo essas mais prejudicadas pelos 
ventos de cauda do que as aeronaves leves. Segundo Ayres Jr. et al.(2011) as aeronaves com motores a pistão não são consideradas no modelo, pois essas aeronaves estão caindo em desuso.

\subsection{Aeroportos hub e não hub, destinos internacionais}

O aeroporto hub é caracterizado por conectar diferentes voos, permitindo a transferência de passageiros - conexão até seu destino final. Como costumam ainda oferecer voos internacionais, as etapas percorridas são usualmente maiores que em aeroportos menores, sendo assim, o cansaço da tripulação pode acometer em risco nas operações. Outro ponto é a maior oferta e demanda de voos que pode gerar mais atrasos nas operações, necessitando de uma gestão mais complexa do aeroporto e do tráfego aéreo (Andersson et al., 2001). Logo, a tecnologia e a segurança são ostensivamente empregadas, de modo a amenizar possíveis perigos. No modelo, essa dualidade de risco e segurança acaba refletindo nos coeficientes. Apesar das operações serem mais complexas, aumentando o perigo de incidentes/acidentes, os esforços para que os pousos e as decolagens sejam realizados com sucesso são maiores. Já os aeroportos que não são hubs e que não oferecem voos internacionais, têm pistas de menor comprimento do que os aeroportos de grande porte, logo, haverá um aumento na probabilidade de ultrapassagem dos limites da pista no pouso - LDOR.

\subsection{Fator de criticidade}

O fator de criticidade é um elemento de ponderação entre o comprimento da pista no aeroporto analisado e a distância necessária requerida por uma aeronave. Valores positivos representam situações onde a distância disponível é menor que a requerida, aumentando o risco de acidentes. Quanto maior o valor, mais crítica é a operação, pois a margem de segurança decresce. A variável é obtida através da razão entre a distância requerida, no aeroporto, pela condição de máximo peso de decolagem - MTOW e a distância disponível declarada. No modelo os coeficientes com maior magnitude são dessa variável, ou seja, o risco aumentará para qualquer tipo de evento caso não haja o comprimento de pista necessário para a aeronave efetuar a operação, principalmente na possibilidade de ultrapassagem no pouso - LDOR. Aeroportos regionais que possuem comprimento de pista nessas condições, e que operam eventualmente aeronaves de maior porte, podem enfrentar maior probabilidade de uma saída de pista.

\section{METODOLOGIA}

Para analisar o comportamento das operações da aviação regional, o Aeroporto de Joinville foi escolhido como estudo de caso. Para calcular a probabilidade de ocorrência de um incidente (Equação 1) foram obtidos os dados operacionais - dia e horário da operação, modelo da aeronave, tipo de operação - pouso ou decolagem, cabeceira que foi efetuada a operação, do aeroporto analisado através de um pedido formal junto ao DECEA - Departamento de Controle do Espaço Aéreo, via ICEA - Instituto de Controle do Espaço Aéreo. Foram obtidos também os dados meteorológicos - temperatura, nível de teto, visibilidade horizontal, direção e intensidade do vento etc., do aeroporto nos mesmos dias e horários das operações efetuadas, através do METAR - Meteorological Aerodrome Report (REDEMET, 2014). As operações analisadas e dados obtidos datam de 01 de fevereiro de 2013 a 28 de fevereiro de 2014. Os dados operacionais poderiam ter sido tomados no HOTRAN - Horário de Transporte (ANAC, 2015), porém como nessa base de dados existe apenas a aviação regular, não seriam obtidos os dados da aviação geral e o estudo ficaria descaracterizado. 
O Aeroporto Lauro Carneiro de Loyola está localizado no norte do estado de Santa Catarina. De acordo com o CGNA - Centro de Gerenciamento da Navegação Aérea (2013), em 2013, 55\% das operações foram de aviação geral, $43 \%$ comerciais e $2 \%$ militares. Ainda de acordo com o CGNA (2013), os usuários do transporte aéreo nesse aeroporto são normalmente empresários e executivos. A grande movimentação de operações de aeronaves menores indica um cunho regional do aeroporto. Além de ser um aeroporto regional, possui como característica climática elevados níveis de precipitação e frequente condição de neblina, tendo sido em 2012 o aeroporto que mais fechou, dentre àqueles gerenciados pela Infraero. Por se tratar de um aeroporto litorâneo, as operações sofrem ainda influência dos ventos, principalmente quando estes são transversais à pista. Logo, utilizá-lo como objeto de análise de risco significa considerar o extremo dos casos dos aeroportos regionais brasileiros, em relação às condições climáticas. Além disso, atualmente a faixa de pista do aeroporto em questão é invadida pelo pátio de aeronaves e pelas taxiways, o que caracteriza uma não conformidade. 0 modelo possibilita o cálculo da probabilidade de colisão com tais obstáculos e a suas possíveis consequências, no entanto, como o foco do estudo está nas dimensões da faixa de pista, tal análise não foi realizada.

Conforme recomendado no IAC 154 -1001 (DAC, 2004), a referência para o risco de uma determinada situação de perigo em um aeródromo é de $1.10^{-7}$, ou seja, um evento a cada 10 milhões de operações. Logo, a média da probabilidade de todos os resultados obtidos através da Equação 3, para cada um dos cinco tipos de eventos, deverá ter esse nível de segurança. Assim, para determinação da área livre de obstáculos, deve-se primeiramente calcular as probabilidades de alguma aeronave exceder os limites das faixas de pista atual. Posteriormente, verificamse novas configurações para a faixa de pista, garantindo a segurança preconizada. Isto é feito por meio do cálculo da média das probabilidades de saída de pista, e da probabilidade de a aeronave exceder determinadas distâncias para cada operação efetuada no aeródromo no período analisado. Este é um procedimento repetitivo, o qual deve ser feito até que se encontrem as distâncias da pista que condizem ao nível de segurança exigido. Tais distâncias correspondem à área livre de obstáculo que deve ser reservada para caso a aeronave saia dos limites da pista, essa área é conhecida como faixa de pista. A configuração da atual faixa de pista do Aeroporto Lauro Carneiro de Loyola é apresentada na Figura 1.

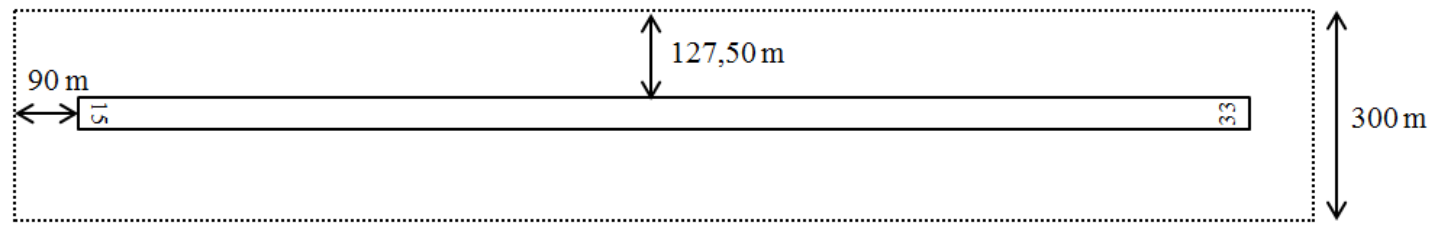

Figura 1. Geometria atual da faixa de pista do Aeroporto de Joinville

Como o modelo ACRP 50 (Ayres Jr. et al.,2011) não considera as aeronaves com motores a pistão, todas as aeronaves desse tipo foram consideradas, a critério de cálculo, como sendo os menores jatos permitidos de acordo com o modelo. Essa consideração usa o pressuposto de que existe uma tendência na substituição das aeronaves que possuem motores a pistão pelas aeronaves a jato. A substituição dos dois tipos de aeronave não descaracteriza as operações de um aeroporto regional, porém pode interferir no resultado final encontrado. Assim, essa é uma consideração a favor da segurança, uma vez que aeronaves a jato usualmente necessitam de maior comprimento de pista que as aeronaves com motores a pistão, devido ao seu maior peso máximo de decolagem. 


\section{RESULTADOS E DISCUSSÕES}

\subsection{Configuração geométrica atual}

Antes de considerar hipóteses em relação a alterações nas dimensões da faixa de pista, foram analisadas as condições atuais do Aeroporto de Joinville, tal como demonstrado na Figura 1. Na Figura 2 são apresentados diagramas de caixa - box plot ou box and whisker, para cada tipo de evento. Os diagramas foram criados a partir dos dados coletados, como explicitado na seção anterior, e aplicados no modelo ACRP 50, calculando a possibilidade de a aeronave sair para além dos limites da atual faixa de pista. Esses diagramas apresentam a frequência das operações de acordo com os intervalos de probabilidade de ocorrência dos eventos. Através desse tipo de gráfico é possível identificar a centralidade dos resultados, dispersão, desvio da simetria e possíveis observações atípicas - outliers (Montgomery e Runger, 2014).

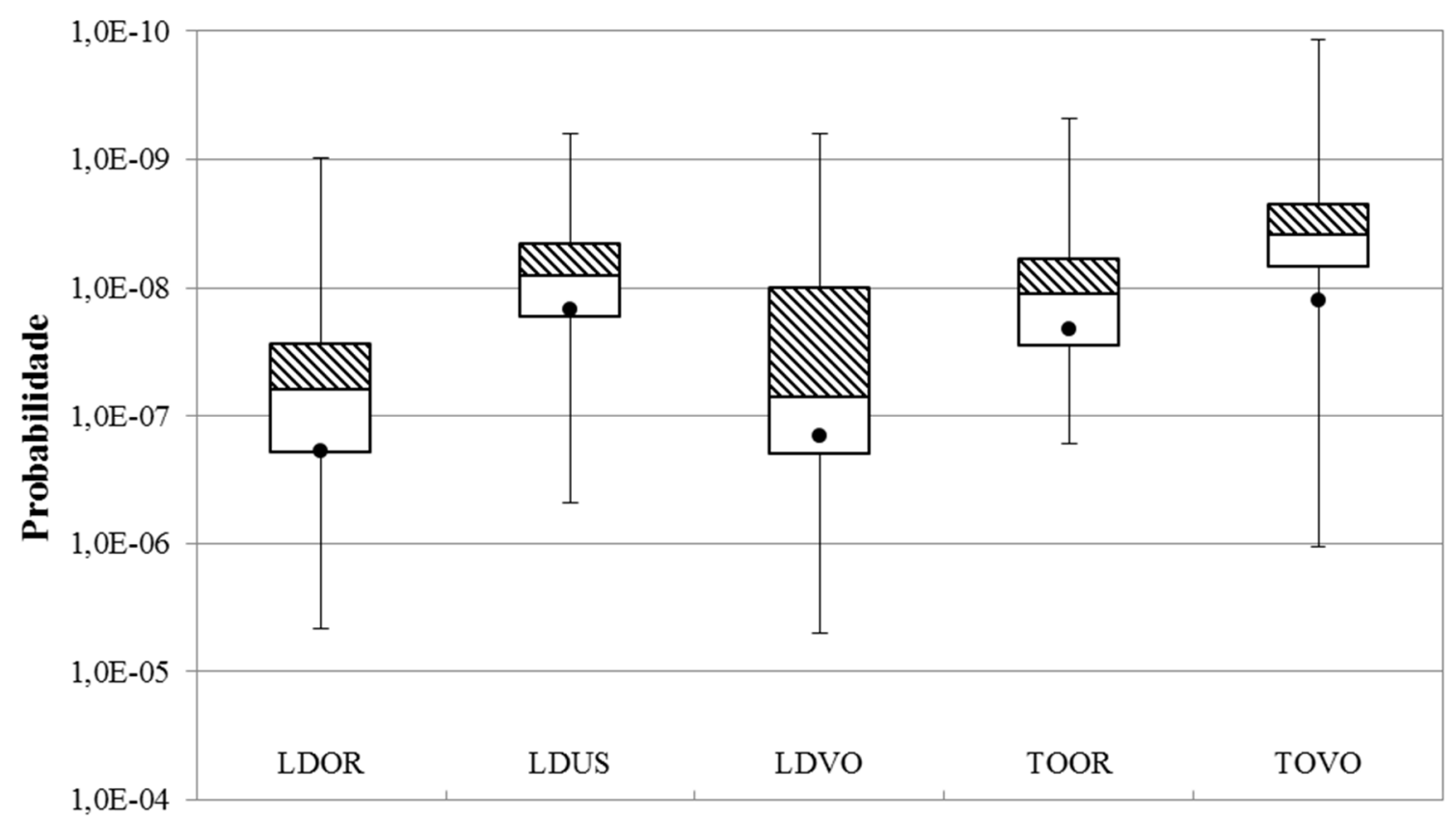

Figura 2. Diagrama de caixa da probabilidade de ocorrência de eventos no Aeroporto de Joinville, considerando a geometria do aeródromo durante o intervalo amostral considerado.

Pelos diagramas pode-se afirmar que a ultrapassagem no pouso (LDOR) e a saída lateral no pouso (LDVO) são os eventos mais prováveis de acontecer, considerando a atual dimensão da faixa de pista. E o evento mais improvável é a saída lateral na decolagem (TOVO). Outro fator importante na análise do diagrama de caixa é a dispersão das probabilidades, que pode ser vista através da amplitude - range no primeiro, segundo e terceiro quartil. A amplitude é a diferença entre o valor máximo e mínimo, sendo possível observar que o evento com maior multiplicidade de probabilidades é a saída lateral no pouso - LDVO. Essa maior dispersão das probabilidades se deve a quantidade superior de variáveis atribuídas a esse evento, criando, portanto, maior variabilidade de situações. Os pontos nos diagramas de caixa são as médias da distribuição de probabilidades de cada evento. Apesar das distribuições serem diferentes, as médias dos eventos LDUS, TOOR e TOVO são valores próximos, da mesma forma que os eventos LDOR e LDVO.

A alta probabilidade de ocorrência de ultrapassagem no pouso - LDOR pode ter tido como causa a variável "Aeroporto $h u b /$ não $h u b$ ", pois diferentemente dos demais eventos, onde ela é inexistente ou favorável à segurança, essa variável apresenta um coeficiente que favorece o risco (devido ao aeroporto ser classificado como não hub essa variável irá aparecer no cálculo de todas as operações). Além disso, no pouso, $65 \%$ das operações foram realizadas por aeronaves classificadas como D/E/F, ou seja, de pequeno porte - de acordo com as especificações do 
modelo. Conforme apontado no Item 3.1, a falta de experiência dos pilotos de aeronaves menores pode aumentar a probabilidade de erros humanos acontecerem. A frequente presença desses coeficientes nos cálculos, e principalmente, a junção deles nas operações fez com que a possibilidade de ultrapassagem dos limites da pista no pouso aumentasse.

Já no desvio lateral na decolagem - TOVO aconteceu o contrário. Não apenas a presença de fatores que aumentam o risco foi menor, como também houve maior participação de fatores que diminuem a probabilidade de acontecer um evento - sinal negativo no coeficiente. Um exemplo disso é que $89 \%$ das operações de decolagem aconteceram dentro do intervalo de 4 a 8 milhas náuticas de visibilidade horizontal, obtendo um coeficiente favorável a segurança. Nesse mesmo evento, a ocorrência de temperatura acima de $15^{\circ} \mathrm{C}$ e abaixo de $25^{\circ} \mathrm{C}$ não influenciou a operação, pois esse intervalo de temperatura é a referência do modelo. Exceto quando a temperatura foi menor que $5^{\circ} \mathrm{C}$ na operação, tendo acontecido apenas em uma decolagem, aproximadamente $0 \%$ da amostra, é que houve o aumento do risco. Nos demais casos, 39\%, a segurança cresceu devido aos demais coeficientes do modelo.

\subsection{Variações da geometria da faixa de pista}

Após analisar a configuração atual da faixa de pista do Aeroporto de Joinville foram exploradas outras dimensões para a faixa de pista, além daquela já existente no aeródromo. De forma a encontrar uma região com a segurança desejada, fixou-se um valor de Y determinando a probabilidade de ocorrência dos eventos para diversos valores de "X", sendo o nível de segurança de referência igual a $1.10^{-7}$.

Na Figura 3 são apresentados os primeiros resultados desses cálculos. Nesse primeiro momento, no caso A para os eventos de overrun e undershoot, foi considerado um Y fixo de 22,5 metros, valor esse correspondente à metade da largura da pista. Tomou-se esse valor para identificar qual seria o comprimento necessário de $\mathrm{X}$ caso a aeronave tivesse apenas a largura da pista para percorrer no caso de uma ultrapassagem - LDOR ou TOOR, ou de um toque antecipado no pouso - LDUS. Esse valor de Y representa a dimensão mínima necessária que deve existir em uma faixa de pista.
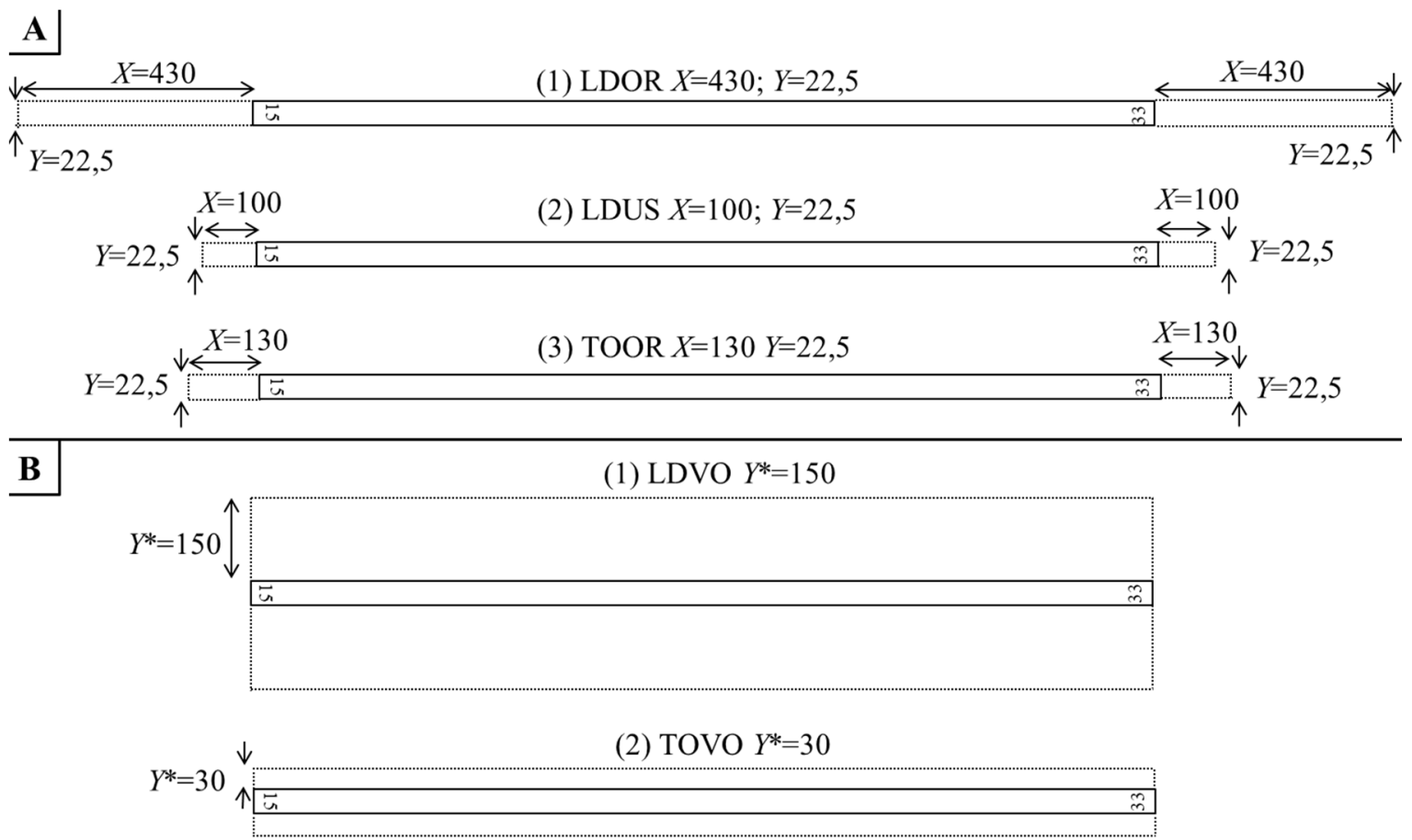

Figura 3. Configurações de faixas de pista quando o $\mathrm{Y}=22,5$ metros - fixo, (A) nos eventos de overrun e undershoot, (B) no caso de veer-off. 
Ainda na Figura 3A são apresentadas três configurações de faixa de pista, ultrapassagem no pouso (1) e na decolagem (3), e toque antecipado no pouso (2). Como já havia sido apontado anteriormente na Figura 2, o evento mais provável de ocorrer é a ultrapassagem no pouso LDOR. Sendo assim, é necessário mais área de faixa de pista para tornar a operação segura, pois o resultado encontrado significa que a probabilidade da aeronave exceder qualquer uma dessas dimensões é de $1.10^{-7}$, como o previsto. Os resultados do LDUS (2) e do TOOR (3) são próximos, e apesar dos valores de $\mathrm{X}$ serem praticamente quatro vezes menores do que o do calculado para o LDOR (1), eles ainda são maiores do que a dimensão encontrada para o eixo X na faixa de pista do Aeroporto de Joinville - 90 metros. Esses resultados para X foram maiores que o esperado, pois eles compensam o valor considerado para o Y - 22,5 metros.

Na Figura 3B pode-se observar que foi necessário aumentar o comprimento lateral da faixa de pista, ultrapassando os 127,5 metros atuais (a partir da borda da pista), para conseguir a taxa de segurança necessária. Também chama a atenção, nas configurações da Figura 3B, a diferença de resultados no pouso (1) e na decolagem (2), apesar de ser decorrente do mesmo tipo de evento.

Após calcular a probabilidade de todos os eventos individualmente, foram utilizados como referência para o cálculo da faixa de pista final os eventos com maior probabilidade de ocorrência, sendo esses a saída lateral no pouso - LDVO e a ultrapassagem na decolagem - LDOR. Sendo assim, o valor encontrado no cálculo do LDVO, $Y^{*}=150$ metros, Figura 3B, foi utilizado como referência para o cálculo final da faixa de pista. Com isso calculou-se novamente o evento mais crítico, a ultrapassagem na decolagem - LDOR, tendo como resultado o valor de 120 metros após o término da pista, e de 172,5 metros a partir do eixo da pista. Esse resultado garante o nível de segurança necessário para todos os eventos calculados, a configuração final fica demonstrada na Figura 4.

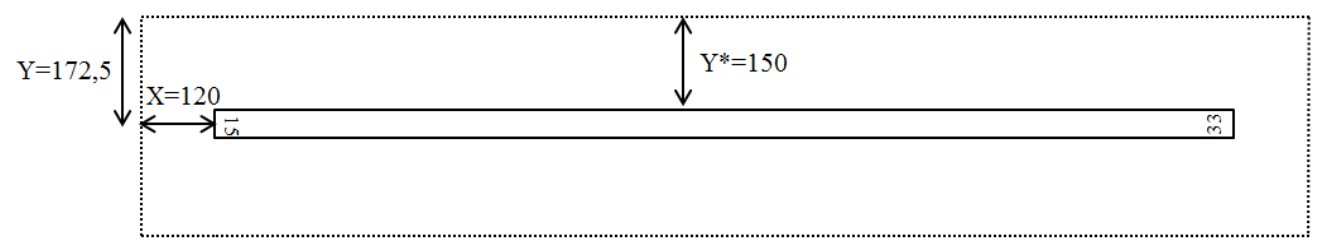

Figura 4. Resultado final da análise da faixa de pista do Aeroporto de Joinville

Tanto no eixo x quanto no eixo y houve aumento dos comprimentos, se comparado à geometria atual do Aeroporto de Joinville. Houve acréscimo de 30 metros no sentido longitudinal, ao final de cada cabeceira, e de 22,5 metros no sentido transversal, a partir da borda da pista. Para os cálculos realizados todas as operações de pouso e decolagem foram consideradas iguais nas duas cabeceiras, deixando a faixa de pista simétrica.

Os resultados indicaram a necessidade de aumento da faixa de pista, tendência essa diferente da esperada, apesar do aeroporto atender aos mesmos requisitos que aeroportos internacionais e de maior movimentação operacional que ele. 0 resultado final calculado é superior à faixa de pista do Aeroporto de Guarulhos no sentido transversal, a qual possui 150 metros a partir do eixo da pista, 22,5 metros a menos do que o apresentado na Figura 4. Isso leva a crer que, mesmo que o modelo não tenha sido criado com um histórico de dados do Brasil, o nível de segurança exigido deveria estar relacionado às características operacionais do aeroporto como, por exemplo, o número de movimento de aeronaves.

\subsection{Gradiente de probabilidades}

Com o objetivo de discutir a possibilidade de aperfeiçoamento da geometria da faixa de pista, analisou-se a variação da média das probabilidades calculadas para a ultrapassagem no pouso 
- LDOR das operações efetuadas nas duas cabeceiras. Foram escolhidas as probabilidades provenientes do cálculo do LDOR, pois em todos os cálculos ele apresentou maior possibilidade de ocorrência, permitindo melhor visualização da discussão proposta.

Na Figura 5 é apresentada metade da pista de Joinville, cabeceira 33, juntamente com o mapa de probabilidades de a aeronave exceder a diagonal resultante dos pontos $X$ e $Y$. Para isso, foram feitos cálculos da probabilidade de LDOR variando as dimensões $X$ e $Y$, sendo a variação a cada 10 metros em $X$ e a cada 22,5 metros em $Y$. Não foram realizados os cálculos para a faixa de pista lateral, em caso de veer-off, pois a probabilidade irá diminuir homogeneamente ao longo da pista, conforme o afastamento da borda da pista.

Primeiramente, é possível observar que o maior nível de risco se concentra na faixa central da pista. A linha traçada no mapa de probabilidade, Figura 5, indica os pontos de $X$ e $Y$ onde a probabilidade de ultrapassagem da aeronave é de $1.10^{-7}$, logo, a área dentro desse traçado não possui a taxa de segurança preconizada. Deve-se salientar que o nível de segurança utilizado implica uma probabilidade de um incidente a cada 10 milhões de operações. Além disso, o cálculo da média não é um indicador de tendência central e nem de predomínio de algum valor. De acordo com Silva e de Barros (2016), o valor do nível de segurança utilizado como referência foi estabelecido na década de 80, e tem sido utilizado desde então, mesmo que, de acordo com os autores, este valor possa variar de acordo com o país e região. Finalmente, Eddowes et al. (2001) afirmam que o nível de segurança deve ser de acordo com a natureza do risco e a possibilidade de redução deste, logo não deve ser tomado para a análise, de todo e qualquer caso, um valor padrão de nível de segurança.

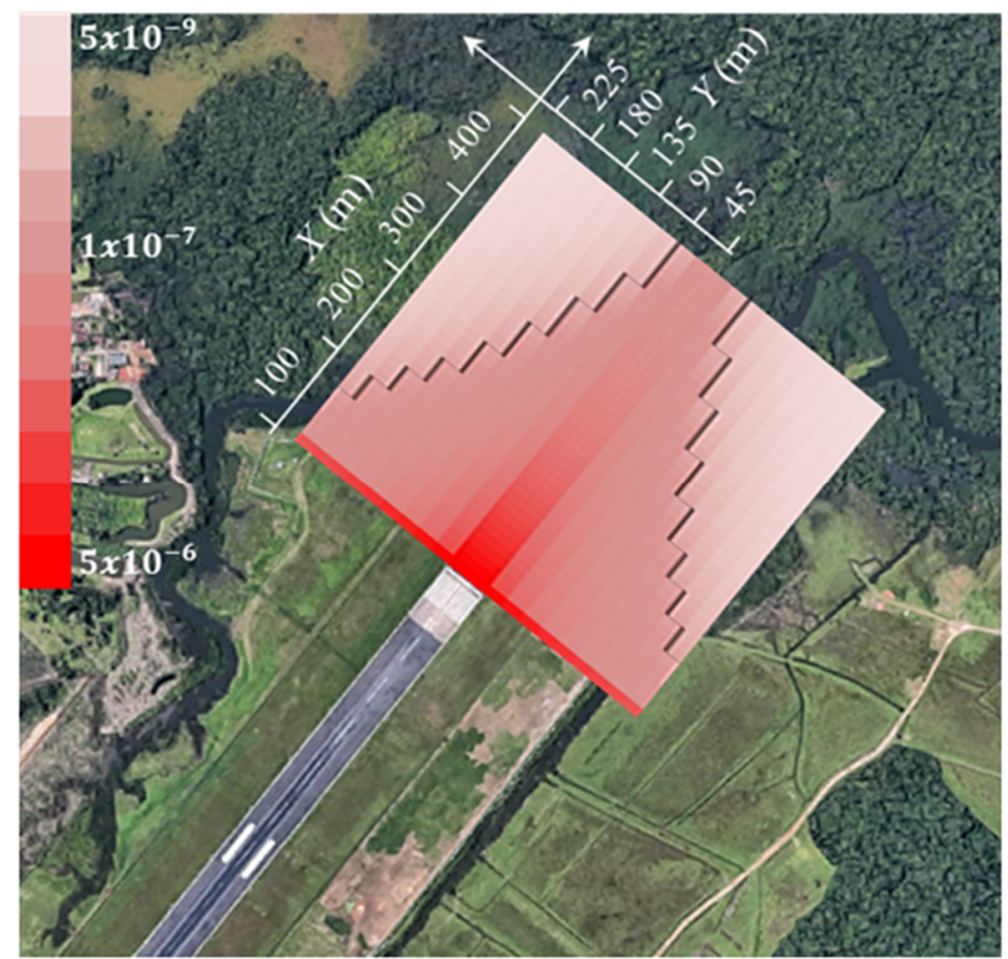

Figura 5. Distribuição de probabilidades para o evento de overrun sobre imagem de satélite no Aeroporto de Joinville (Google maps, 2017).

Em um estudo do Aeroporto Internacional de Los Angeles - LAX, Barnett et al. (2015) concluíram que a probabilidade de uma colisão fatal a cada 200 anos - estimativa de nível de tráfego para 2020, não implicaria uma necessidade prática de aumento da segurança operacional. No que se refere ao Aeroporto de Joinville, a movimentação total de aeronaves em 2014 foi de 12.622 pousos e decolagens (INFRAERO, 2017), sendo essa a maior movimentação dos últimos 
cinco anos, $30 \%$ a mais que 2016 . Considerando então o nível de segurança de $1.10^{-7}$, a probabilidade seria de um incidente a cada 792 anos aproximadamente. Sendo assim, não há necessidade prática de aumentar as dimensões da faixa de pista existente no Aeroporto de Joinville.

Esses resultados confirmam a hipótese de que os níveis de segurança exigidos não são coerentes. Conforme mencionado anteriormente, o Brasil possui um grande número de aeródromos de pequeno porte, os quais possuem movimentação anual ainda menor que a do aeroporto aqui analisado. Sendo assim, não parece plausível que os requisitos de infraestrutura de tais aeródromos sejam os mesmos que os exigidos de aeroportos de grande porte tais como o Aeroporto de São Paulo-Congonhas, com movimentação anual de 213.000 pousos e decolagens, e o Aeroporto do Rio de Janeiro - Santos Dumont, com 105.600 operações por ano (INFRAERO, 2017). Portanto, é necessário um estudo mais aprofundado dos requisitos exigidos para os aeroportos regionais brasileiros, e as consequências advindas de possíveis mudanças nas normas. Um exemplo do efeito da diminuição da faixa de pista seria o deslocamento das superfícies de aproximação. Com essa mudança, o crescimento da cidade se daria mais perto dos limites da pista de pouso e decolagem do aeroporto. Construções, tais como edifícios, anteriormente tidos como não conformidade a certa distância do aeroporto, seriam considerados como condição padrão, diminuindo a segurança operacional. Além dessa, outras consequências devem ser consideradas em estudos mais aprofundados do tema.

\section{CONCLUSÃO}

O modelo de análise de risco utilizado possui limitações, principalmente para a análise da realidade brasileira, sendo uma delas a não consideração de aeronaves com motor a pistão. Além disso, os resultados são balizados pela base histórica de dados pelo qual o modelo foi criado, e pela qual nele é inserido para a efetuação dos cálculos. Sendo assim, os resultados aqui expostos devem ser aproveitados como forma de discussão acerca dos requisitos de projeto para aeroportos regionais e não como valores finais de projeto. Nesse contexto, justifica-se a necessidade de desenvolvimento de um modelo que seja mais adequado à realidade do transporte aéreo no Brasil. Outra possibilidade seria ainda a validação do modelo utilizado para a realidade brasileira, especificamente para aeroportos regionais.

Apesar dos resultados apresentarem a necessidade de aumentar as dimensões da faixa de pista, essa não parece ser uma proposta razoável, tendo em vista que o aeroporto estudado segue as mesmas normas utilizadas por aeroportos internacionais e de movimentação superior a ele. Portanto, com base nos resultados encontrados, a flexibilização dos requisitos da faixa de pista não seria possível. No entanto, estes resultados trazem à tona a necessidade de discussão com relação aos níveis de segurança exigidos. As exigências de infraestrutura hoje adotadas não fazem distinção quanto ao tamanho, movimentação ou tipo de utilização do aeródromo. Assim, aeroportos regionais como o Aeroporto de Joinville, devem atender aos mesmos requisitos que aeródromos como o Aeroporto de Guarulhos ou mesmo o Aeroporto de Los Angeles, 3o em movimentação nos EUA. Portanto, talvez seja mais plausível verificar a possibilidade de flexibilização dos níveis de segurança de aeroportos regionais, uma vez que, devido a menor movimentação, a quantidade de possíveis ocorrências de acidentes é inferior a aeroportos de grande porte.

Discussões aprofundadas do tema devem levar em consideração as consequências de possíveis flexibilizações das normas para aeroportos regionais. Uma consequência da diminuição das dimensões da faixa de pista seria a redução de segurança, devido a alterações nas superfícies de aproximação, de acordo com o crescimento das cidades. Problemas atualmente tratados como não conformidades, em caso de alteração da norma, poderiam ser tratados como regulares. Portanto, essa é uma discussão que possui muitas lacunas que devem ser observadas em novas discussões. 


\section{AGRADECIMENTOS}

Os autores gostariam de agradecer à Capes pelo auxílio financeiro, e ao DECEA - Departamento de Controle do Espaço Aéreo, que através do ICEA - Instituto de Controle do Espaço Aéreo forneceu os dados operacionais para a realização deste trabalho.

\section{REFERENCES}

Aguiar, M.; A. Stolzer e D. D. Boyd (2017) Rates and causes of accidents for general aviation aircraft operating in a mountainous and high elevation terrain environment. Accident Analysis and Prevention, v. 107, p. 195-201. DOI: 10.1016/j.aap.2017.03.017

Almeida, C. A.; J. L. Farias; L. C. B. Santos; F. F. Santos; C. P. C. Azevedo; F. L. Matheus e L. A. Serra (2016) Ocorrências aeronáuticas: Panorama estatístico da aviação brasileira - Aviação civil 2006-2015. Centro de Investigação e Prevenção de Acidentes Aeronáuticos (CENIPA). Brasília, 2016.

ANAC (2009) RBAC 154: Projeto de Aeródromos. Agência Nacional de Aviação Civil, Brasília - DF, 2009.

ANAC (2012) RBAC 61: Licenças, habilitações e certificados para pilotos. Agência Nacional de Aviação Civil, Brasília - DF, 2012.

ANAC, Agência Nacional de Aviação Civil. Hotran - Horário de Transporte. Brasília - DF. <http://www.anac.gov.br/assuntos/dados-e-estatisticas/historico-de-voos> (Janeiro, 2015).

Andersson, K.; F. Carr; W. D. Hall; N. Pujet e E. Feron (2001) Analysis, Modeling, and Control of Ground Operations at Hub Airports, cap. 20. In: Donohue, G. L.; A. G. Zellweger; H. Rediess; C. Pusch (eds.) Air Transportation Systems Engineering. Ed. American Institute of Aeronautics and Astronautics, Inc., Reston, Virginia. DOI: 10.2514/5.9781600866630.0305.0341.

Ayres Jr., M; H. Shirazi; R. Carvalho; J. Hall; R. Speir; E. Arambula; R. David; D. Wong e J. Gadzinski (2011) ACRP Report 50: Improvement Models for Risk Assessment of Runway Safety Areas. TRB, Washington, D.C. DOI: 10.17226/13635.

Bandeira, M. C. G. S. P.; A. R. Correia e M. R. Martins (2017) Method for measuring factors that affect the performance of pilots. Transportes, v. 25, n. 2, p. 156 - 169. DOI: 10.14295/transportes.v25i2.1374.

Barnett, A.; M. Ball; G. Donohue; M. Hansen; A. Odoni e A. Trani (2015) Collision course? The north airfield safety study at Los Angeles International Airport (LAX). Transportation Research Part A, v.77, p. 14 - 34. D0I: 10.1016/j.tra.2015.03.003.

Bazargan, M. e V. S. Guzhva (2011) Impact of gender, age and experience of pilots on general aviation accidents. Accident Analysis and Prevention, v. 43, n. 3, p. 962 - 970. DOI: 10.1016/j.aap.2010.11.023.

CGNA (2013) Anuário estatístico de tráfego aéreo 2013. Centro de Gerenciamento da Navegação Aérea, Rio de Janeiro, 2013.

Correia, A. R. e J. A. Ribeiro Neto (2014) Safety evaluation of runway safety areas: case study at major Brazilian airports. Journal of the Brazilian Air Transportation Research Society, v. 10, n. 1, p. 25-36.

DAC (2004) IAC 154-1001: Termo de referência para estudo aeronáutico. Departamento de Aviação Civil, Rio de Janeiro, 2004.

Donehue, P. e D. Baker (2012) Remote, rural and regional airports in Australia. Transport Policy, v. 24, p. 232-239. DOI: 10.1016/j.tranpol.2012.08.007.

Eddowes, M.; J. Hancox e A. MacInnes (2001) Final report on the risk analysis in support of aerodrome design rules: Report for the Norwegian Civil Aviation Authority. Warrington, UK: AEA Technologies.

Fortes, J. L. de C. e A. R. Correia (2012) Safety assessment at airports: São Paulo/Congonhas airport - A case study. Journal of the Brazilian Air Transportation Research Society, v. 8, n.1, p. 29-42.

Freire, L. L. de A. A flexibilização de requisitos de serviços de salvamento e combate a em aeródromos como mecanismo de fomento à aviação é possível? 2016. 95f. Dissertação (Mestrado em Transportes) - Faculdade de Tecnologia, Universidade de Brasília.

Google maps. Imagem do Aeroporto de Joinville, 2017. <https://www.google.com.br/maps/place/Aeroporto+Lauro+Carneiro+de+Loyola+-+Joinville+JOI/@-26.2232477,48.8031553,1148m/data=!3m1!1e3!4m5!3m4!1s0x94deae80f6c1bb5b:0xbf9a0cbe77a5748f!8m2!3d-26.2234999!4d$48.7998418>$ (Setembro, 2017).

Halpern, N. e S. Bråthen (2011) Impacts of airports on regional accessibility and social development. Journal of Transport Geography, v. 19, n. 6, p. 1145-1154. DOI: 10.1016/j.jtrangeo.2010.11.006.

INFRAERO, Empresa Brasileira de Infraestrutura Aeroportuária. Anuário Estatístico Operacional 2016, 2017. Brasília-DF. <http://www.infraero.gov.br/images/stories/Estatistica/anuario/anuario_2016.pdf> (Setembro, 2017)

INMET, Instituto Nacional de Meteorologia. Glossário, 2015. Brasília - DF. <http://www.inmet.gov.br/portal/index.php?r=home/page\&page=glossario $>$ (Janeiro, 2015).

Montgomery, D. C. e G. C. Runger (2014) Estatística Aplicada e Probabilidade para Engenheiros. 5. ed., Ed. LTC, Rio de Janeiro, RJ.

REDEMET, Rede de Meteorologia do Comando da Aeronáutica. Consulta de mensagens meteorológicas: METAR: 2013-2014. Rio de Janeiro. <https://www.redemet.aer.mil.br/?i=produtos\&p=consulta-de-mensagens-opmet> (Outubro, 2014).

Silva, A. e A. G. de Barros (2016) Quantitative risk evaluation of obstacle limitation surfaces for final approaches at airports. Journal of Aviation Technology and Engineering, v. 5, n. 2, p. 51-68. DOI: 10.7771/2159-6670.1110

Trucco, P., M. De Ambroggi e M. C. Leva (2015) Topological risk mapping of runway overruns: A probabilistic approach. Reliability Engineering and System Safety, v. 142, p. 433-443. DOI: 10.1016/j.ress.2015.06.006.

Valdés, R. M. A.; F. G. Comendador; L. M. Gordún e F. J. Sáez Nieto (2011) The development of probabilistic models to estimate accident risk (due to runway overrun and landing undershoot) applicable to the design and construction of runway safety areas. Safety Science, v. 49, n. 5, p. 633-650. DOI: 10.1016/j.ssci.2010.09.020. 
Wilke, S.; A. Majumdar e W. Y. Ochieng (2015) Modelling runway incursion severity. Accident Analysis and Prevention, v.79, p. 88-99. DOI: 10.1016/j.aap.2015.03.016.

Wooldridge, J. M. (2012) Introductory Econometrics - A Modern Approach (2ํe.) CENGAGE Learning. 